\title{
Quantitative analysis of lung sounds for monitoring idiopathic pulmonary fibrosis: a prospective pilot study
}

\begin{abstract}
To the Editor:
Accurate monitoring of disease progression in idiopathic pulmonary fibrosis (IPF) is crucial for predicting prognosis and optimising management, including the initiation of therapies, the appropriate timing of supportive care and the prompt referral for lung transplantation. While forced vital capacity (FVC) via spirometry has been accepted as the most feasible and reliable tool to assess the deterioration in these patients in clinical practice as well as in randomised controlled trials $[1,2]$, in recent years both visual evaluation and computer-based analysis of high-resolution computed tomography (HRCT) parenchymal patterns have been shown to correlate with disease severity based on FVC and predict outcome in several fibrotic lung diseases, including IPF [3-5]. Electronic stethoscopes, which permit digital recording of lung sounds, potentially represent a simple, noninvasive and reproducible tool for evaluating disease severity and progression in patients with IPF. Although computerised lung sound analysis can discriminate between crackles due to pulmonary fibrosis and those due to pneumonia or heart failure [6-8], the utility of lung sounds for monitoring the course of the disease in these patients is still unknown. We have recently identified that typical "Velcro-type" crackles are closely correlated with the extent of several HRCT features, suggesting that lung sounds might facilitate the early detection of fibrotic lung disease [9]. In this prospective pilot study, we evaluated the longitudinal change in acoustic features of lung sounds recorded from patients with IPF and validated such features by examining correlations with several clinical, physiological and radiological parameters.
\end{abstract}

Patients with a diagnosis of IPF were consecutively recruited from those attending the interstitial lung disease (ILD) clinic of the University Hospital of Southampton (UK) between March and September 2015. A group of healthy volunteers were also enrolled as controls.

Patients attended visits every 2 months for a total of seven study appointments over 12 months of clinical observation. At each visit, lung sounds were recorded consecutively over 10 different chest locations identified according to the guidelines for computerised respiratory sounds analysis (CORSA) [10], using a digital stethoscope (Littmann 3200; 3M, St. Paul, MN, USA) held manually. Other serial measurements included lung volumes via spirometry and diffusing capacity of the lung for carbon monoxide ( $D \mathrm{LCO}$ ), dyspnoea and quality of life (assessed via the University of California San Diego Shortness of Breath Questionnaire (UCSD-SOB) and St George's Respiratory Questionnaire (SGRQ)), and tolerance to exercise (6-min walk test (6MWT)). A HRCT scan was performed at baseline if not performed within 12 months prior to screening, and a follow-up chest HRCT scan was performed at the end of the study (12 months of follow-up). Consecutive HRCT scans were visually scored by two expert thoracic radiologists (A.R.L. and N.S.) for extent of ILD abnormalities and extent of individual radiological features. Volumes for parenchymal features were also automatically quantified using the Computer-Aided Lung Informatics for Pathology Evaluation and Rating (CALIPER) software (Mayo Clinic, Rochester, MN, USA). Lung sound data was analysed via acoustic signals processing algorithms written in Matlab (version 2015a; MathWorks, Natick, MA, USA) [11]. For each sound file 481 variables corresponding to distinct acoustic features were generated, ranging from statistical properties to features related to the energy content of the signal and to the frequency domain.

@ERSpublications

Quantitative analysis of lung sounds may represent a tool for monitoring patients with idiopathic pulmonary fibrosis http://ow.ly/9ICq30naWk6

Cite this article as: Sgalla G, Larici AR, Sverzellati N, et al. Quantitative analysis of lung sounds for monitoring idiopathic pulmonary fibrosis: a prospective pilot study. Eur Respir J 2019; 53: 1802093 [https://doi.org/10.1183/13993003.02093-2018]. 
The intraclass correlation coefficient (ICC) was used to assess intra-subject reproducibility of acoustic features [12]. Multivariate ANOVA was used to compare acoustic features between IPF and control subjects. Repeated measures ANOVA was used to assess longitudinal change in the selected parameters. Estimated differences of means were calculated after adjusting for age, sex, body mass index (BMI) and recording site, as applicable. Multivariate linear regression was performed to investigate the correlations between acoustic features and other parameters measured at different time-points throughout the study. All data was entered into the SPSS software package (version 24, IBM Corp., Armonk, NY, USA).

19 patients were enrolled in the study: three (15.7\%) died during the observation period due to progressive disease and two (10.5\%) others withdrew due to poor health which prevented further clinical visits. Mean $\pm \mathrm{SD}$ age was $70.8 \pm 6.53$ years and there was a male predominance (84.2\%). More than two-thirds of patients $(68.4 \%)$ were receiving antifibrotic treatment at baseline. At baseline the study population had a moderate functional impairment (mean predicted FVC: 73.7\%) and reduced DLCO (mean predicted DLCO: $43.5 \%)$. The study population showed a trend towards a decline in per cent predicted FVC $(-5.9 \%, 95 \%$ CI $-4.27-16.08, \mathrm{p}=0.862)$ and per cent predicted DLCO $(-5.5 \%, 95 \%$ CI $-2.34-13.34, \mathrm{p}=0.35)$ over 12 months of clinical observation.

An intra-subject reliability analysis using three repeated recordings from four IPF subjects identified a set of 19 reproducible acoustic features (a cut-off of ICC $>0.5$ was arbitrarily chosen for indicating acceptable reliability). The combination of the 19 acoustic features was shown to discriminate IPF subjects from the healthy volunteers $(\mathrm{F}=11.837, \mathrm{p}<0.001)$. Six features out of 19 underwent significant change between baseline and the end of the study and were therefore used for correlation analysis. Under multivariate linear regression analysis, the set of acoustic features were more associated with visual scores of total ILD extent (adjusted $\mathrm{R}^{2}=0.106, \mathrm{p}<0.005$ ) and CALIPER-measured ILD total and peripheral volumes (adjusted $\mathrm{R}^{2}=0.116$ and 0.136 , respectively, $\mathrm{p}<0.001$ ) than with physiological parameters such as FVC (adjusted $\mathrm{R}^{2}=0.031$, $\mathrm{p}<0.001$ ) or 6 -min walking distance (adjusted $\mathrm{R}^{2}=0.065 \mathrm{p}<0.001$ ). As for the relationships between lung sounds features and individual radiological abnormalities, the strongest associations were found with reticulation and honeycombing, either visually assessed $\left(\mathrm{R}^{2}=0.043, \mathrm{p}=0.04\right.$ and $\mathrm{R}^{2}=0.093, \mathrm{p}=0.001$, respectively) or quantified via CALIPER $\left(\mathrm{R}^{2}=0.108, \mathrm{p}<0.001\right.$ and $\mathrm{R}^{2}=0.094, \mathrm{p}=0.001$, respectively). The association between lung sounds and fibrotic patterns on HRCT was further assessed against the Composite Physiologic Index (CPI), a strong predictor of morphological severity of disease and mortality in IPF [13]. A model built combining the acoustic features and CPI performed better at discriminating extensive fibrosis on HRCT, adjudicated as sum of honeycombing and reticulation $\geqslant 50 \%$, as compared to CPI alone (area under the curve (AUC) $=0.79$ (95\% CI 0.69-0.89) and AUC=0.69 (95\% CI 0.58-0.79), respectively) (figure 1).

In this pilot study, we identified a set of acoustic features of lung sounds recorded from IPF patients and showed them to be reproducible, change over time and be associated with established measures of disease severity and clinical deterioration.

FIGURE 1 Receiver operating characteristic curves of the different models evaluated in the study for identification of extent of fibrosis $\geqslant 50 \%$ on high-resolution computed tomography scan. Extent of fibrosis was calculated as the sum of honeycombing + reticulation visual percentage scores. Acoustic features: area under the curve (AUC) $=0.73$ $(95 \% \mathrm{Cl}$ 0.63-0.84); composite physiologic index (CPI): $A U C=0.69$ (95\% Cl 0.58-0.79); acoustic features $+\mathrm{CPI}: \mathrm{AUC}=0.79(95 \% \mathrm{Cl} 0.69-0.89)$.

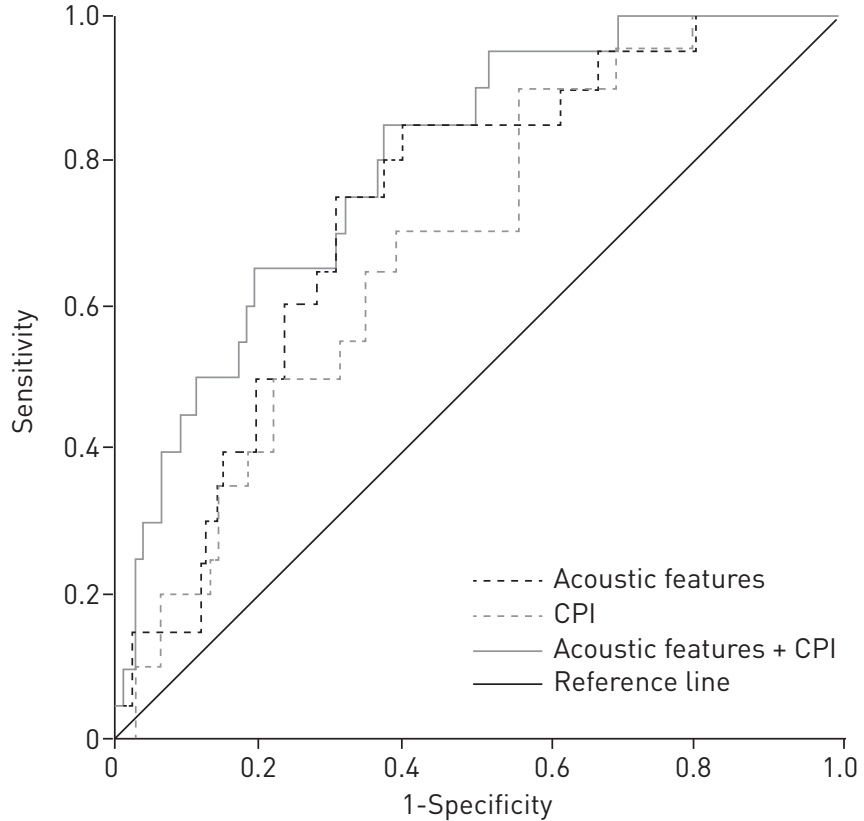


Chest auscultation is a simple, point-of-care, noninvasive and inexpensive assessment routinely used for monitoring the course and the response to treatment of respiratory disorders. Automated analysis of lung sounds was initially studied more than 30 years ago; however, most studies have focused on the characterisation and automatic classification of diverse adventitious lung sounds rather than their longitudinal behaviour. A key preliminary finding in our study is that serial digital measurements of lung sounds using a simple electronic stethoscope provide reproducible and clinically significant acoustic measures in IPF. The analysis of longitudinal acoustic data in this study demonstrates, for the first time, that a set of reproducible features undergo significant change over a 12-month observation period, making them a promising surrogate of disease progression in IPF. On multivariate regression analysis, the selected acoustic features showed the strongest relationships with the extent of ILD on HRCT, either visually scored or quantitatively estimated using CALIPER. Reticulation and honeycombing were most strongly associated with acoustic features, putatively suggesting they make a major contribution to the character of "Velcro-type" crackles. The link between acoustic features and disease morphology on HRCT was corroborated by the finding that combining the CPI with acoustic features resulted in a model that was more accurate at identifying extensive fibrosis on HRCT than the CPI alone.

Our study has several strengths. It was prospective in design and used a standardised, reproducible method for lung sound recording. We collected a broad set of acoustic features and evaluated relationships between individual features and both semiquantitative (visual) and quantitative (CALIPER) measurements of disease extent. Lastly, we used commercially available electronic stethoscopes, which are inexpensive and readily available in most parts of the world. A limitation of this study is that it involved a small population from a single centre, which limited its power to explore if change in lung sounds can actually predict disease progression or survival in these patients. Nevertheless, these pilot data provide the basis for a larger multicentre trial aimed at assessing the utility of lung sounds as a biomarker of disease behaviour in patients with IPF.

In conclusion, in this study we showed, for the first time, that acoustic features of lung sounds are a reproducible and valid metric of disease severity in patients with IPF. In principle, quantitative lung sounds analysis is a feasible, point-of-care, inexpensive and noninvasive novel clinical biomarker in IPF. The further exploration of lung sounds in larger longitudinal cohorts, such as those of clinical trials, could clarify their potential role for monitoring disease progression in these patients. Any effort in this direction should be supported by the development of novel tools providing easily interpretable read-outs for clinicians.

Giacomo Sgalla $^{1,2}$, Anna Rita Larici ${ }^{1}$, Nicola Sverzellati ${ }^{3}$, Brian Bartholmai ${ }^{4}$, Simon L.F. Walsh ${ }^{5}$, Dragana Nikolic ${ }^{6}$, Anna Barney ${ }^{6}$, Sophie Fletcher ${ }^{2}$, Mark Jones ${ }^{2}$, Donna D Davies ${ }^{2}$ and Luca Richeldi ${ }^{1,2}$

${ }^{1}$ Fondazione Policlinico Universitario A. Gemelli IRCCS, Università Cattolica del Sacro Cuore, Rome, Italy. ${ }^{2}$ NIHR Southampton Biomedical Research Centre and Clinical and Experimental Sciences, University of Southampton, Southampton, UK. ${ }^{3}$ Dept of Radiology, University Hospital of Parma, Parma, Italy. ${ }^{4}$ Dept of Radiology, Mayo Clinic, Rochester, MN, USA. ${ }^{5}$ Dept of Radiology, King's College Hospital, London, UK. ${ }^{6}$ Institute for Sound and Vibration Research, University of Southampton, Southampton, UK.

Correspondence: Giacomo Sgalla, Unità Operativa Complessa di Pneumologia, Fondazione Policlinico Universitario A. Gemelli IRCCS, Università Cattolica del Sacro Cuore, Largo A. Gemelli 8, 00168 Rome, Italy. E-mail: giacomo. sgalla@guest.policlinicogemelli.it

Received: Sept 032018 | Accepted after revision: Nov 142018

Conflict of interest: G. Sgalla reports personal fees from Boehringer Ingelheim, outside the submitted work. A.R. Larici has nothing to disclose. N. Sverzellati reports personal fees from Roche and Boehringer Ingelheim, outside the submitted work. B. Bartholmai reports grants from NIH/NHLBI (he is Principal Investigator of the NHLBI Lung Tissue Research Consortium; Mayo Clinic receives funds for this project and B. Bartholmai's effort), and personal fees from Promedior, LLC (for acting as a scientific advisor) and from Imbio, LLC (Mayo Clinic and B. Bartholmai receive fees/royalties from Imbio, LLC for the license of CALIPER analysis software), outside the submitted work. In addition, B. Bartholmai has a patent "systems and methods for analyzing in vivo tissue volumes using medical imaging" pending (which is relevant to the CALIPER functionality and visualisation of quantitative results). S.L.F. Walsh reports personal fees from Boehringer Ingelheim (speaker and consultancy fees), Roche (speaker and consultancy fees), Intermune (speaker fees), Bracco (speaker fees), and Sanofi-Genzyme (consultancy fees), outside the submitted work. D. Nikolic has nothing to disclose. A. Barney has nothing to disclose. S. Fletcher reports funding to attend conferences, speaker fees and fees for advisory boards from Roche and Boehringer Ingelheim. M. Jones has nothing to disclose. D.D. Davies reports she is a founder and shareholder of Synairgen and has received personal fees from Synairgen (consultant), outside the submitted work. L. Richeldi reports personal fees and other from Boehringer Ingelheim (trial principal investigator), grants and personal fees from InterMune, personal fees from Cipla and Vertex, and other from AstraZeneca, GlaxoSmithKline, Sanofi-Aventis, Celgene, Prometic, Roche and Takeda (scientific advisory boards), during the conduct of the study.

Support statement: The conduct of the study was supported by funding from the NIHR Southampton Respiratory Biomedical Research Unit (UK). Littmann 3200 electronic stethoscopes were provided by $3 \mathrm{M}$ through an educational grant. Funding information for this article has been deposited with the Crossref Funder Registry. 


\section{References}

1 Du Bois RM, Weycker D, Albera C, et al. Forced vital capacity in patients with idiopathic pulmonary fibrosis: test properties and minimal clinically important difference. Am J Respir Crit Care Med 2011; 184: 1382-1389.

2 Richeldi L, Ryerson CJ, Lee JS, et al. Relative versus absolute change in forced vital capacity in idiopathic pulmonary fibrosis. Thorax 2012; 67: 407-411.

3 Jacob J, Bartholmai BJ, Rajagopalan S, et al. Automated quantitative computed tomography versus visual computed tomography scoring in idiopathic pulmonary fibrosis. J Thorac Imaging 2016; 31: 304-311.

4 Jacob J, Bartholmai BJ, Rajagopalan S, et al. Mortality prediction in idiopathic pulmonary fibrosis: evaluation of computer-based CT analysis with conventional severity measures. Eur Respir J 2017; 49: 1601011.

5 Jacob J, Bartholmai BJ, Rajagopalan S, et al. Predicting outcomes in idiopathic pulmonary fibrosis using automated computed tomographic analysis. Am J Respir Crit Care Med 2018; 198: 767-776.

6 Ponte DF, Moraes R, Hizume DC, et al. Characterization of crackles from patients with fibrosis, heart failure and pneumonia. Med Eng Phys 2013; 35: 448-456.

7 Flietstra B, Markuzon N, Vyshedskiy A, et al. Automated analysis of crackles in patients with interstitial pulmonary fibrosis. Pulm Med 2011; 2011: 590506.

8 Piirilä P, Sovijärvi ARA. Crackles: recording, analysis and clinical significance. Eur Respir J 1995; 8: 2139-2148.

9 Sgalla G, Walsh SLF, Sverzellati N, et al. "Velcro-type" crackles predict specific radiologic features of fibrotic interstitial lung disease. BMC Pulm Med 2018; 18: 103.

10 Sovijärvi ARA, Vanderschoot J, Earis JE, eds. Computerized respiratory sound analysis (CORSA): recommended standards for terms and techniques. ERS Task Force Report. Eur Respir Rev 2000; 10: 585-649.

11 Lartillot O, Toiviainen P. A matlab toolbox for musical feature extraction from audio. Proc. of the 10th Int. Conference on Digital Audio Effects (DAFx-07), Bordeaux, France, September 10-15, 2007. http://dafx.labri.fr/ main/papers/p237.pdf

12 Fleiss JL. Reliability of measurement. The Design and Analysis of Clinical Experiments. John Wiley \& Sons, Inc., 2011: 1-32. http://doi.wiley.com/10.1002/9781118032923.ch1

13 Wells AU, Desai SR, Rubens MB, et al. Idiopathic pulmonary fibrosis: a composite physiologic index derived from disease extent observed by computed tomography. Am J Respir Crit Care Med 2003; 167: 962-969. 